\title{
Dynamic control for synchronization of separated cortical areas through thalamic relay
}

\author{
Leonardo L. Gollo \\ IFISC, Instituto de Fúsica Interdisciplinar y Sistemas Complejos (CSIC-UIB), \\ Campus Universitat des Illes Balears, \\ E-07122 Palma de Mallorca, Spain \\ Claudio Mirasso* \\ IFISC, Instituto de Física Interdisciplinar y Sistemas Complejos (CSIC-UIB), \\ Campus Universitat des Illes Balears, \\ E-07122 Palma de Mallorca, Spain \\ Alessandro E. P. Villa \\ NeuroHeuristic Research Group \\ http://www.neuroheuristic.org \\ Grenoble Institut des Neurosciences (GIN) UMR_S 836 INSERM, \\ Université Joseph Fourier, Grenoble, France \\ and \\ Information Systems Department ISI, \\ University of Lausanne, Switzerland
}

(Dated: September 1, 2009) 


\begin{abstract}
Binding of features and information which are processed at different cortical areas is generally supposed to be achieved by synchrony despite the non-negligible delays between the cortical areas. In this work we study the dynamics and synchronization properties of a simplified model of the thalamocortical circuit where different cortical areas are interconnected with a certain delay, that is longer than the internal time scale of the neurons. Using this simple model we find that the thalamus could serve as a central subcortical area that is able to generate zero-lag synchrony between distant cortical areas by means of dynamical relaying [1]. Our results show that the model circuit is able to generate fast oscillations in frequency ranges like beta and gamma bands triggered by an external input to the thalamus formed by independent Poisson trains. We propose a control mechanism to turn "On" and "Off" the synchronization between cortical areas by simply changing the relative rate of the external input fed into dorsal and ventral thalamic neuronal populations. The current results emphasize the hypothesis that the thalamus could control the dynamics of the thalamocortical functional networks enabling two separated cortical areas to be either synchronized (at zero-lag) or unsynchronized. This control may happen at a fast time scale in agreement with experimental data and without any need of plasticity or adaptation mechanisms which typically require longer time scales.
\end{abstract}

Keywords: dynamic relaying, thalamocortical cirduit, zero lag synchronization, correlation, firing pattern, thalamus, reticular thalamic nucleus

*Electronic address: claudio@ifisc.uib-csic.es; corresponding author 


\section{Introduction}

In the central nervous system (CNS) it is assumed that the information is mainly represented by the activity of neurons transmitted to other neurons through synaptic links. The extent of the neural network activated by a specific "piece of information" is a never ending matter of investigation but it is accepted that both average levels of discharges (firing rate) [2] and precise spike timing contribute to neural coding. Spatiotemporal firing patterns $[3,4]$ and coherent oscillatory neural activity [5] associated to sensory and behavioral events support the hypothesis that temporal information plays a key role in brain processing. Empirical phenomena and extensive experimental data validated across different species [6-9] emphasize the importance of emerging cortico-cortical synchrony as a major phenomenon for binding features distributed neural activity [10-12]. Despite the success of physical models to reproduce oscillatory patterns of neural activity it is not clear whether the synchronization is the result of network processing exclusively limited to cortico-cortical interactions or subcortical structures might also intervene [13, 14].

The thalamus is a structure of CNS that could play an important role to let the emergence or to control cortico-cortical synchronization because the exchange of information between the thalamus and cerebral cortex is a general feature of all ascending sensory pathways but olfaction $[15,16]$. The connectivity pattern between thalamus and cortex is usually viewed as been characterized by thalamocortical integration and corticothalamic feedback [17-19]. Multiple thalamocortical modules characterized by the same basic connectivity may be assumed to work in parallel and include three main components (see Fig. 1): (i) dorsal thalamic neurons (e.g. from the medial geniculate body for the auditory pathway or from the lateral geniculate body for the visual pathway) recipient of the sensory input from the periphery; (ii) cells of the thalamic reticular nucleus (RE), a major component of the ventral thalamus; (iii) the cortical area receiving the corresponding thalamic input. RE receives collateral inputs from both thalamocortical and corticothalamic fibres and sends its inhibitory projections to the dorsal thalamus, thus regulating the firing mode of the thalamocortical neurons. RE receives inputs also from several forebrain and midbrain areas known to exert modulatory functions [20], in particular from nerve growth factor responsive basal forebrain cholinergic cells [21] that are involved in many cognitive functions and whose dysfunction is associated to Alzheimer's Disease. In the auditory system evidence exist that corticofugal 
activity regulates the response properties of thalamic cell assemblies by changing their bandwidth responsiveness to pure tones [22] thus allowing to selectively extract information from the incoming sensory signals according to the cortical activity [18]. This model suggests that the thalamocortical circuit carries embedded features that enable the build-up of combined supervised and unsupervised information processing akin to produce an adaptive filter [23].

The current study is not aimed at simulating any detailed thalamocortical circuit, but rather to assess the role of simple variables that could play a major role in controlling the emergence and maintenance of synchronized activity in distributed cortical areas that project to the same thalamic nuclei. Our model predicts that small changes in the cortical neurons firing rate, due to non-correlated background synaptic activity in the thalamic region, is capable of generating single or multi-frequency oscillations along with zero-lag synchronization between distant cortical regions. We quantify this synchronized state by measuring the signal-to-noise ratio which does not monotonically increase with the firing rate. According to our model, thalamic activity plays a key role in controlling the appearance of lag free synchronization between cortical areas. In addition, despite its oversimplification, the model provides hints about the conditions necessary to achieve that synchronization. We report an efficient control set as the ratio of dorsal over ventral thalamus external input activity to switch on thalamocortical synchronous dynamics. That switch occurs at a fast time scale, without any need of synaptic plasticity which would require longer time scales [11]. The type of control that we suggest is not limited to an "On"- "Off" switch, but it allows to control the appearance of synchronous activity over an extended range of frequencies despite the delays involved in the long-range cortico-cortical interactions [1, 24-26].

\section{Methods}

To study the synchronization of cortical activity facilitated by the thalamic relay we conducted extensive numerical simulations of a reduced thalamocortical model of spiking integrate and fire neurons subject to background noise and an external driving. The model

includes both local synapses and long-range interactions with different delays according to functional connectivity in a four populations motif. [27]. The simulations were performed using NEST (the neuronal simulation tool) [28, 29]. 

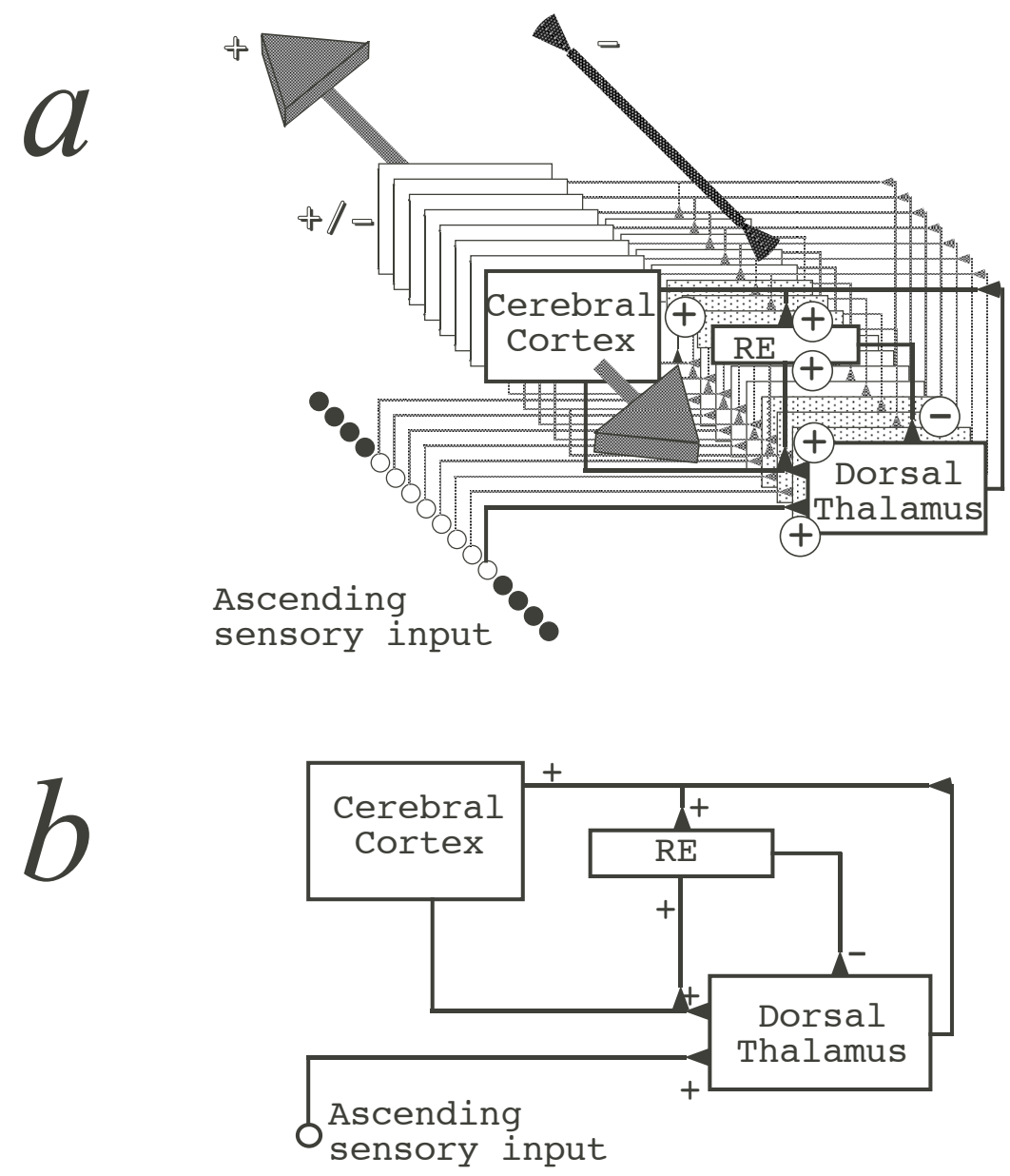

FIG. 1: a: A functional scheme of the modular organisation of the thalamocortical auditory pathway. The signs indicate the nature of the connections, $(+)$ excitatory and (-) inhibitory. RE: reticular nucleus of the thalamus. Note the excitatory input from the ascending sensory pathway to the dorsal thalamus, the excitatory projection from the thalamus to the cortex with a collateral to RE, and the excitatory projection of the cortex to the thalamus with a collateral to RE. The only output of RE is an inhibitory backprojection to the thalamus. Other intrinsic connections exist within RE (mainly inhibitory) as well as within cortex and thalamus. b: Explicit connections within one thalamocortical module.

Neuronal model. The integrate-and-fire neuron model [30] for each neuron $i$ satisfies the following dynamical equation for the membrane potential $V_{i}(t)$ :

$$
\tau_{m e m}(m) \frac{d V_{i}(t)}{d t}=-V_{i}(t)+R I_{i}(t)
$$

Where $\tau_{\text {mem }}(m)$ is the membrane time constant of neuron $i$ belonging to the population $m$ (as in Fig. 2); $I_{i}(t)$ is the total current arriving to the soma. The last term in the above 
equation is given by the sum of all postsynaptic potentials (PSP) of neurons belonging to the network plus the total postsynaptic potentials of all external neurons, the latter being modeled as a Poisson process. Thus,

$$
R I_{i}(t)=\tau_{m e m}(m) \sum_{j} J(j) \sum_{k} \delta\left(t-t_{j}^{k}-\tau(z, m)\right)+V_{e x t}
$$

The first sum is taken over all the presynaptic neuron $j$, each neuron receives $C_{e}(m, z)$ excitatory synapses and $C_{i}(m, z)$ inhibitory synapses and they depend on the inter-population (long-range) connections $z$ if both neurons belong to different populations or otherwise on the population $m$ to whom they belong. $t_{j}^{k}$ is the time of the $k-t h$ spike received by neuron $i$ from its neighbor $j$. The axonal conduction delay is given by $\tau(z, m)$, which corresponds to a spike of a presynaptic neuron $j$ that reaches neuron $i$. $J(j)$ stands for the PSP and depends on whether its presynaptic neighbor neuron $j$ is excitatory $\left(J(j)=J_{e}\right)$ or inhibitory $\left(J(j)=J_{i}\right) . V_{\text {ext }}$ is the postsynaptic potential generated by neurons from outside the thalamocortical network. It is given by an independent and homogeneous Poisson process of $N_{\text {ext }}$ external neurons, each one firing with a fixed average rate $\nu(m)$. The external spike contributes with a change of the membrane potential by $J_{\text {ext }}$ when it impinges upon neuron $i$. The dynamics of the neurons can be described as following: the neurons start at a rest potential $V_{r}(m)$ which can be changed by the synaptic current. If the potential $V_{i}(t)$ of the $i$-th neuron reaches the threshold $\theta(m)$ this neuron fires and its potential relaxes back to $V_{r}(m)$ with a certain refractory period $\left(\tau_{r p}=2 \mathrm{~ms}\right)$.

In the reduced thalamocortical system, each population $m$ is described by different parameters, as summarized in Table I.

Thalamocortical model. The thalamocortical model was built to check whether the thalamus can act as a relay for a zero-lag cortical synchrony to occur. The thalamus was considered as two separate populations, one of excitatory thalamocortical relay neurons $(\mathrm{T})$ and another of inhibitory relay neurons corresponding to the thalamic reticular and perigeniculate nuclei $(\mathrm{R})$. Consequently, the topology of our model is given by four populations $[31,32], \mathrm{R}, \mathrm{T}$ and two balanced cortical areas $\left(\mathrm{C}_{1}\right.$ and $\left.\mathrm{C}_{2}\right)$ which may or may not be directly connected. In this way the thalamus is considered as the two central populations that create a recurrent intrathalamic circuit through extensive synaptic connections, as depicted in Fig. 2. It is a hierarchical network, with both an intra population random structure and a simple inter-population pattern of connectivity with longer delays. The populations have 


\begin{tabular}{|l|l|l|l|l|}
\hline$m$ & $\mathrm{C}_{1}, \mathrm{C}_{2}$ & $\mathrm{R}$ & $\mathrm{T}$ & population \\
\hline$N_{e}(m)$ & 800 & 0 & 200 & \# of excitatory neurons \\
$N_{i}(m)$ & 200 & 40 & 0 & \# of inhibitory neurons \\
$\tau_{m e m}(m)$ & $20 \mathrm{~ms}$ & $25 \mathrm{~ms}$ & $15 \mathrm{~ms}$ & membrane time constant \\
$\theta(m)$ & $20.5 \mathrm{mV}$ & $24.65 \mathrm{mV}$ & $15 \mathrm{mV}$ & threshold value \\
$\tau_{r p}$ & $2 \mathrm{~ms}$ & $2 \mathrm{~ms}$ & $2 \mathrm{~ms}$ & refractory period \\
$V_{r}(m)$ & $10 \mathrm{mV}$ & $12.5 \mathrm{mV}$ & $7.5 \mathrm{mV}$ & membrane rest potential \\
$C_{e}(m)$ & 80 & 0 & 5 & \# of excitatory synapses* \\
$C_{i}(m)$ & 20 & 10 & 0 & \# of inhibitory synapses* \\
$\tau(m)$ & $1.5 \mathrm{~ms}$ & $2 \mathrm{~ms}$ & $1 \mathrm{~ms}$ & synaptic delay \\
$J_{e}$ & $0.05 \mathrm{mV}$ & 0 & $0.05 \mathrm{mV}$ & excitatory synaptic efficacy \\
$J_{i}$ & $-0.2 \mathrm{mV}$ & $-0.2 \mathrm{mV}$ & 0 & inhibitory synaptic efficacy \\
\hline
\end{tabular}

TABLE I: Neuronal parameters for the neurons in population $\mathrm{m}$. ${ }^{*}$ Each neuron receives from a neighbor of the same population.

both internal and external connectivity according to the anatomical knowledge of the circuit. Assuming that the thalamus is composed by both $\mathrm{R}$ and $\mathrm{T}$ populations, the thalamocortical model can also be reduced to a three populations network formed by a central thalamic regions $(\mathrm{T}+\mathrm{R})$ and two balanced cortical areas. Each neuron of a given population receives the same amount of postsynaptic connections. The presynaptic neuron are set randomly, therefore, the postsynaptic distribution is binomial for each type of neuron (excitatory or inhibitory) within a given population.

The topology satisfies the following constrains, as shown in Fig. 2: Both $\mathrm{R}\left(C_{C R}\right)$ and $\mathrm{T}\left(C_{C T}\right)$ populations receive cortical feedback, the cortical populations are innervated by $\mathrm{T}\left(C_{T C}\right)$ but do not receive inhibitory feedback from $\mathrm{R}$. There are also direct connection from $\mathrm{R}$ to $\mathrm{T}\left(C_{R T}\right)$ and from $\mathrm{T}$ to $\mathrm{R}\left(C_{T R}\right)$. Finally, we consider long range cortico-cortical connections $\left(C_{C C}\right)$. The number of projecting synapses of each type a given neuron receives (in the corresponding postsynaptic region) and its respective delays for the inter-population connectivity are shown in Table II.

Background activity and external input. To model the background activity we assume that each neuron in the network is connected with $N_{\text {ext }}$ excitatory external neurons 


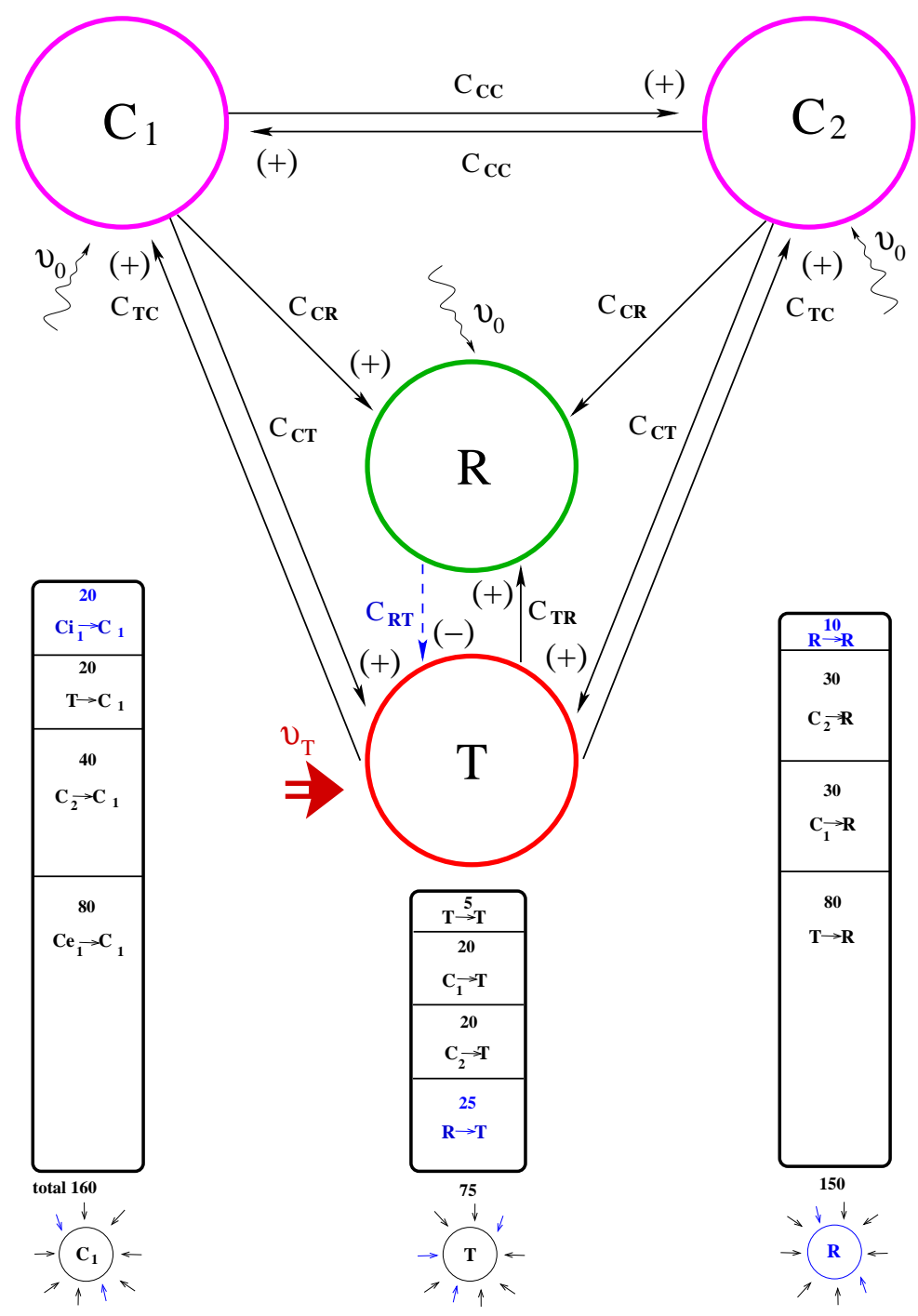

FIG. 2: (color online) Thalamocortical connectivity. The populations are randomly connected. The two cortical ones are balanced with both excitatory (80\%) and inhibitory (20\%) neurons. The parameters which define the neuronal model are presented in Table I. All populations are sparse with exception of $\mathrm{R}$. The thalamus can be considered as both $\mathrm{R}$ and $\mathrm{T}$ together. The inter-population connectivity is described by the parameters of Table II, the dashed blue arrow $\left(C_{R T}\right)$ means that it is inhibitory and black arrows stand for excitatory connections. Our results are robust even when there is no direct cortical connection $\left(C_{C C}\right)$. The background noise and the external driving consists of independent Poisson train impinging in each neuron with parameters of Table III. Neurons in $\mathrm{T}$ are externally driven at rate $\nu_{T}$ meanwhile the other ones receive background activity at rate $\nu_{0}$. The external input is uncorrelated and defines the key parameter: $\frac{\nu_{T}}{\nu_{0}}$. A scheme of all the synaptic inputs innervated in each neuron of each population is presented at the bottom panel. 


\begin{tabular}{|l|l|l|l|l|l|l|l|}
\hline$z$ & $\mathrm{CR}$ & $\mathrm{CT}$ & $\mathrm{TC}$ & $\mathrm{RT}$ & $\mathrm{TR}$ & $\mathrm{CC}$ & inter-population connection \\
\hline$C_{e}(z)$ & 30 & 20 & 20 & 0 & 80 & $0-110$ & \# of excitatory synapses* \\
$C_{i}(z)$ & 0 & 0 & 0 & 25 & 0 & 0 & \# of inhibitory synapses* \\
$\tau(z)$ & $8 \mathrm{~ms}$ & $8 \mathrm{~ms}$ & $5 \mathrm{~ms}$ & $2 \mathrm{~ms}$ & $2 \mathrm{~ms}$ & $5 \mathrm{~ms}$ & synaptic delay \\
\hline
\end{tabular}

TABLE II: Inter-population connectivity parameters. ${ }^{*}$ Each neuron of the second population of $z$ receives from a neighbor of the first population.

\begin{tabular}{|l|l|l|}
\hline$J_{\text {ext }}$ & $0.1 \mathrm{mV}$ & external synaptic efficacy \\
$\nu_{0}$ & $10.0 \mathrm{~Hz}$ & external driving Poisson mean rate \\
& & innervated into $\mathrm{C}$ and $\mathrm{R}$ \\
$\nu_{T}$ & $8.0-45.0 \mathrm{~Hz}$ & external driving Poisson process \\
& mean rate innervated into T \\
$N_{\text {ext }}$ & 450 & number of external neighbors \\
\hline
\end{tabular}

TABLE III: Synaptic efficacy, Poisson external driving and background activity parameters.

subject to an independent random Poisson processes with average rate $\nu_{0}$ for neurons of all the regions. The thalamic region $(\mathrm{T})$ is the only region which receives the external input plus the background activity combined of $N_{\text {ext }}$ excitatory neurons also modeled by independent Poisson process with average rate of $\nu_{T}$. The parameters used for the Poisson background activity and the external driving noise are presented in Table III.

Cross-correlation analysis. We run extensive simulations and analyze the spike trains over several trials. In order to quantify the results from the numerical simulations, we define two values from the cross-correlogram: a) its mean value representing the "noise" level quantifying the expected number of coincidences by chance; b) the peak of the corticocortical cross-correlogram (typically at zero-lag) that stands for the "signal". Those quantities are used to compute the signal-to-noise ratio for different values of $\nu_{T}$ and different strengths of cortical interconnectivity $\left(C_{C C}\right)$. The results are averaged over 100 trials during 2, $000 \mathrm{~ms}$ in a stationary regime after $500 \mathrm{~ms}$ of transient dynamics. The averaged result is condensed in a single cross-correlogram, which measures the mean number of coincidences (in a $2 \mathrm{~ms}$ bin) of 3,000 randomly selected neuron pairs belonging to different populations 
and also averaged over the trials. This procedure allows us to assess the mean behavior of the dynamics and eliminate single trial fluctuations.

The "noise" is determined by the mean over the time lag in the averaged crosscorrelogram. It can also be calculated analytically considering the activity of the two populations just as been independent. Let $F(p)$ be the mean firing rate of population $p$ and $b$ the bin size of the computed cross-correlogram, therefore the mean cross-correlogram (noise) of two arbitrary populations $i$ and $j$ is given by $\left\langle X C O R_{i-j}\right\rangle=F(i) F(j) b$. For a typical thalamocortical circuit the two cortical areas have either maximum synchrony at zero-lag or no synchrony (unless $C_{C C}$ is greater than the number of internal excitatory cortical connections $C_{e C}$ ). Thus the "signal" of the cortico-cortical dynamics is defined as the value it has at zero time lag.

\section{Results}

We model large populations of interacting neurons with delayed connections. To this end, we use the simple integrate and fire (I\&F) neuronal model in order to keep the problem more tractable. It is capable to reproduce the desired behavior under the presence of an incoming signal below its threshold. Eventually, the membrane potential reaches the threshold and fires a pulse. After the pulse is fired, the membrane potential is reset and evolves to its rest potential value with a refractory period $(2 \mathrm{~ms})$. Due to the generation of this spike all postsynaptic neighbor neurons receive an excitatory or inhibitory postsynaptic potential which varies according to the presynaptic neuron type. The process occurs with a corresponding delay depending on the connection. Large delays are associated with inter-population connections and short delays with internal random connections within each population. In the following results, obtained from extensive numerical simulations, we extract the firing rate, cross-correlation indicators, oscillation and synchronization information from the spikes trains of individual neurons and neuron populations. It is worth mentioning that the neuron spike times are reproduced in a quite reliable way, despite the fact that due to its simplicity the model is unable to properly describe the pulse dynamics.

Thalamocortical circuit dynamics. In the most symmetrical case, the $\mathrm{T}$ region is set in order to receive external driving with the same rate as the other populations $\left(\nu_{T}=\nu_{0}\right)$. 
The firing rate in $\mathrm{R}$ is higher than in the cortex which is also higher than in $\mathrm{T}$. Independently of the cortico-cortical interaction $\mathrm{C}_{C C}$, due to the network connectivity and the difference in the neuronal parameters, there is no correlation among the different areas, and the activity is random and irregular. For $\nu_{T}>\nu_{0}$ other scenario takes place. The raster plots of 150 neurons randomly chosen among all neuronal populations illustrate the network dynamics. Such a typical raster plot is depicted in Fig. 3a. It shows the case in which the corticocortical connections are set as $\mathrm{C}_{C C}=40$ and the thalamus is receiving an external input of mean rate $\nu_{T}=7 / 3 \nu_{0}$. The neurons within the populations $\mathrm{T}$ and $\mathrm{R}$ are synchronized at a high frequency. The two cortical areas exhibit a large number of coincidences at zero-lag, meaning that they are synchronized and in-phase. The cross-correlograms (see methods section for details) between the cortical areas and between the thalamus and one cortical area are shown in Fig. 3b, c. The graphic clearly indicates in-phase correlation among cortical areas while the thalamus and the cortical area are out of phase (with the cortical area delayed by $6 \mathrm{~ms}$ ).

The synchronization of the cortical regions depends on the external input to T. Fig. 4 shows the raster plot of a single trial characterized at $t=50 \mathrm{~ms}$ by a sudden increase of the $\mathrm{T}$ activity from the mean rate $\nu_{0}$ to $7 / 3 \nu_{0}$. The synchrony does not occur in the system for low values of input $\nu_{T}$, for instance $\nu_{T}=\nu_{0}$, from $0 \mathrm{~ms}$ to $50 \mathrm{~ms}$ or after the input is switched off, say for time $t>250 \mathrm{~ms}$.

The mean firing rate of $\mathrm{T}, \mathrm{C}$, and $\mathrm{R}$ neurons, computed over 2, $000 \mathrm{~ms}$, increases monotonically as a function input rate $\left(\nu_{T}\right)$ (Fig. 5a). The dependency of the cortical oscillation frequency as a function of $\nu_{T} / \nu_{0}$ is shown in Fig. $5 \mathrm{~b}$ for directly interconnected $\left(C_{C C}=40\right)$ and disconnected $\left(C_{C C}=0\right)$ cortical areas. The frequencies are determined from the power spectrum analysis of the cross correlograms. Only those components whose power is larger than $20 \%$ of the maximum power are considered here. In the disconnected case, the cortical areas oscillate at a single frequency close to the thalamic firing rate (see rate in Fig. 5a). In the interconnected case $\left(C_{C C}=40\right)$ a single frequency dominates the oscillatory dynamics only if $\nu_{T}<2 \nu_{0}$. Beyond this threshold at least two frequencies of oscillation appear. For $\nu_{T}=\frac{7}{3} \nu_{0}$ three different frequencies are observed (as in Fig. 3b). The lowest frequency is related to the firing rate of the neurons within the cortical areas. The intermediate frequency is related to the thalamic firing rate like in the disconnected case. An increase of the oscillatory frequency in the cortical areas is due to greater interaction between the cortex and 

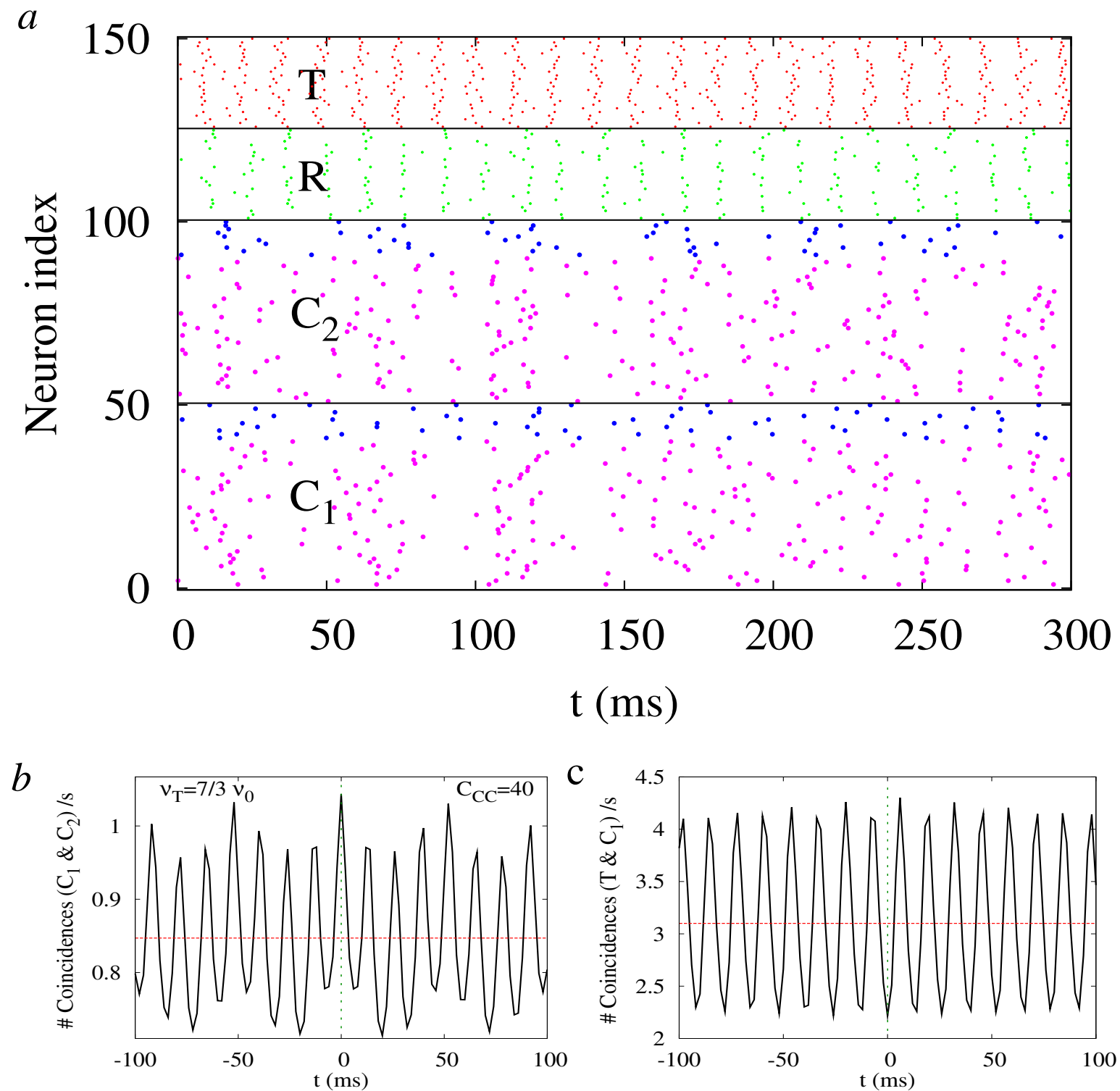

FIG. 3: (color on line) Thalamocortical dynamics. Panel (a) shows raster plots of 150 neurons randomly chosen (50 from each cortical population and 25 neurons from $\mathrm{R}$ and $\mathrm{T}$ ) of the thalamocortical circuit of Fig. 2 in a case that $\mathrm{R}$ and the cortex populations receive external stimulus of $\nu_{0} \mathrm{~Hz}$. The rate in $\mathrm{T}$ is $\nu_{T}=\frac{7}{3} \nu_{0}$. The average cross-correlogram over 100 trials of 3,000 random neuron pairs of different populations with bin size $2 \mathrm{~ms}$ are presented in panels (b) for $\mathrm{C}_{1}$ and $\mathrm{C}_{2}$ areas and (c) for $\mathrm{T}$ and $\mathrm{C}_{1}$. The horizontal dashed line is the mean cross-correlogram value (noise) and the $\mathrm{C}_{1}-\mathrm{C}_{2}$ peak at zero-lag stands for the signal, see text for details. The vertical point line is set at zero-lag only to guide the eye. The maximun of $\mathrm{C}_{1}-\mathrm{C}_{2}$ crosscorrelation occur exactly at zero-lag while the maximum of $\mathrm{T}-\mathrm{C}_{1}$ happen at $6 \mathrm{~ms}$. 


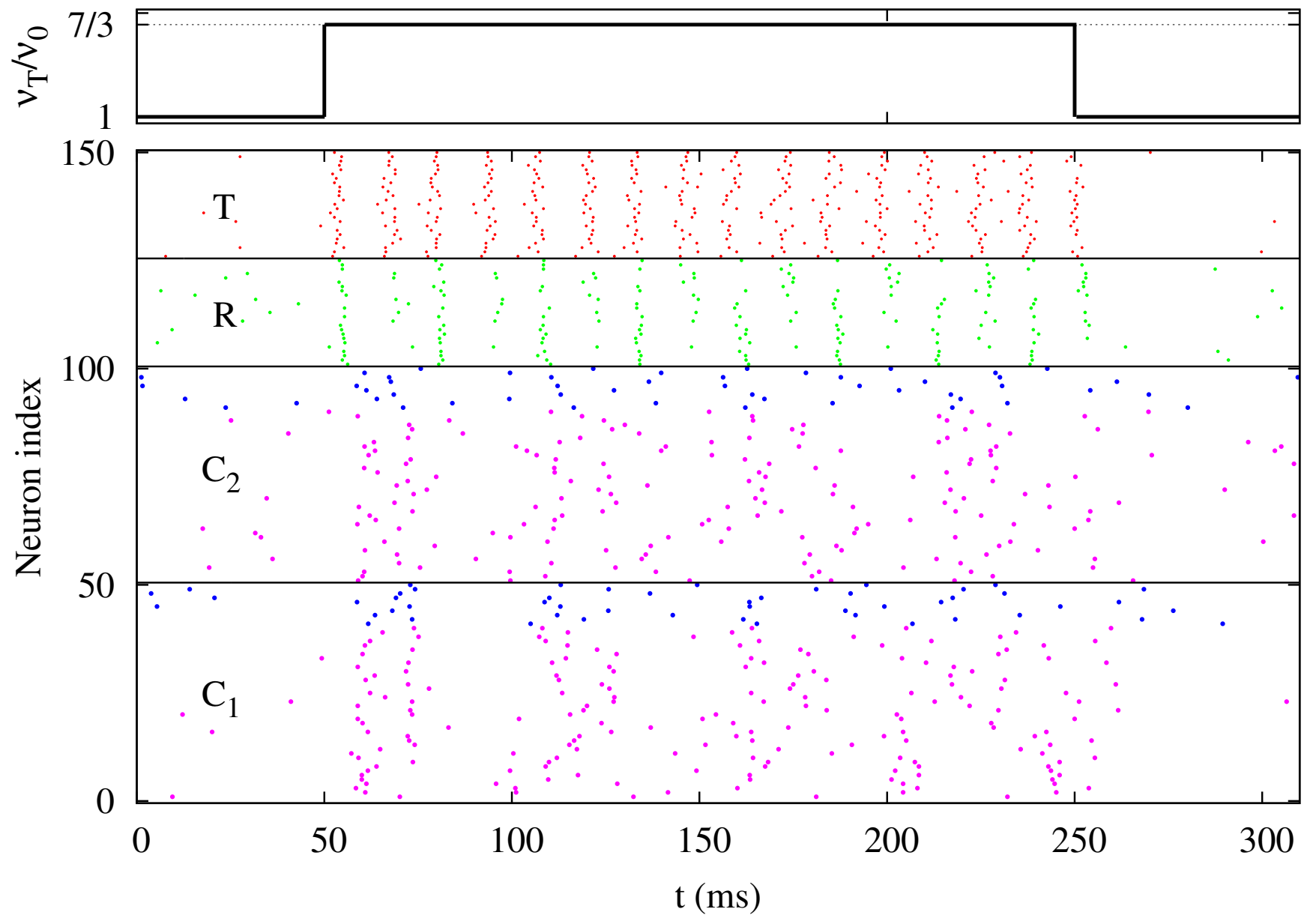

FIG. 4: (color on line) On-off synchronization example. In the cortical areas, the spikes in magenta (blue) stand for excitatory (inhibitory) neurons. The spikes of neurons in $\mathrm{R}$ are in green and those of $\mathrm{T}$ in red. The cortico-cortical connection is set as $C_{C C}=40$.

the thalamus as a function of a larger input fed into the thalamus. The highest frequency component in the interconnected case $\left(C_{C C}=40\right)$ is likely to be related to the inverse of the delay time of the cortico-cortical connection. However, this frequency component is observed only for a very small range of input values.

The signal-to-noise ratio, as defined in the Methods section from the cross-correlograms, as a function of $\nu_{T} / \nu_{0}$ is illustrated in Fig. 5c. The firing rate and the "signal" increase monotonically with the external rate of the input, but interestingly the signal-to-noise function is characterized by a local maximum for uncoupled cortical areas as well as for coupled cortico-cortical areas with connectivity $C_{C C}=40$. The signal-to-noise was quite flat for low values of $\nu_{T}$, then increases until reaching the local maximum. After decreasing from 

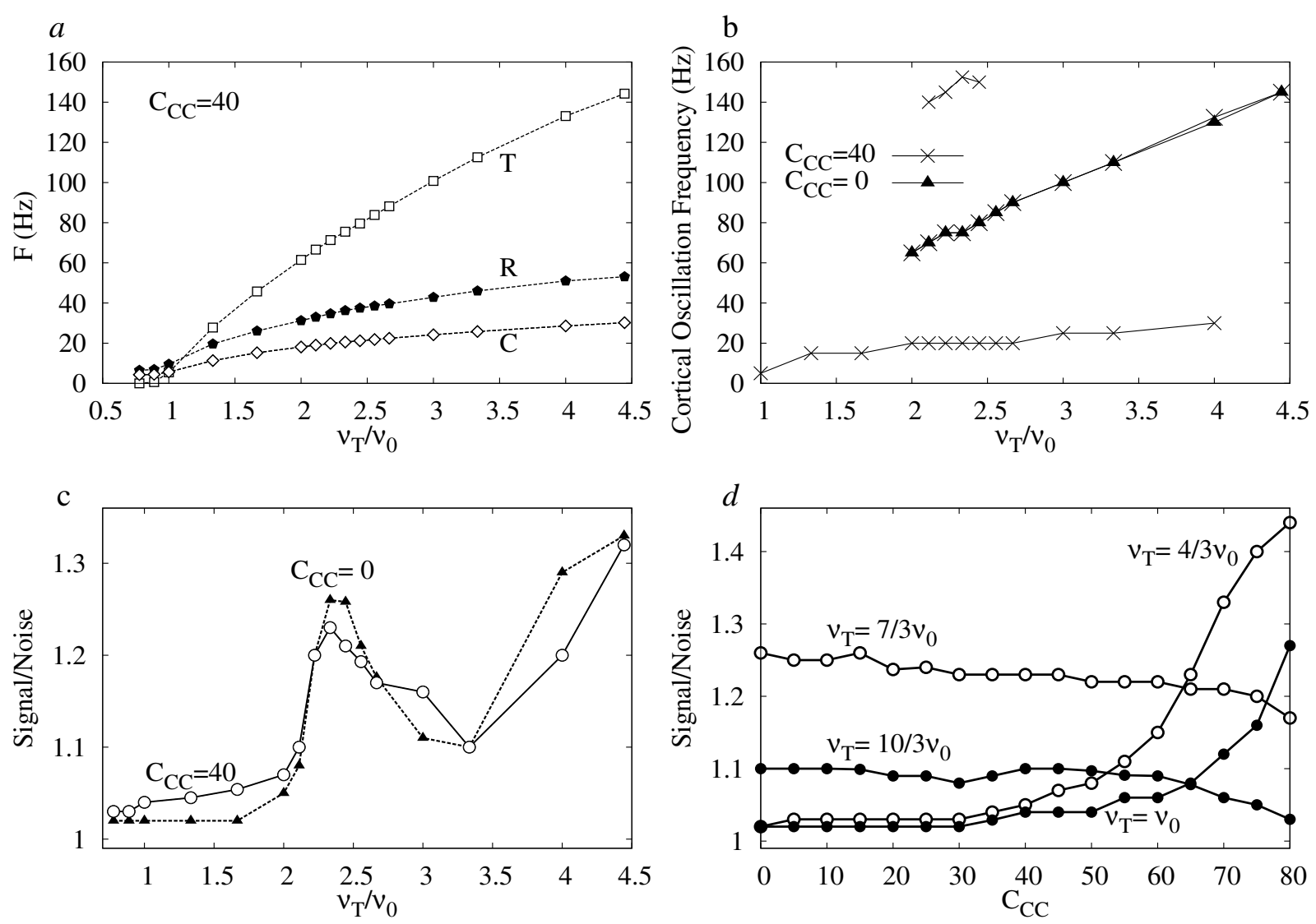

FIG. 5: Unveiling the dynamics - 100 trials analysis. (a): the mean firing rate as a function of the external input $\nu_{T}$ incoming into $\mathrm{T}$ population. In panel (b) we plot the cortical oscillation frequency for increasing $\nu_{T}$ for the case of coupled and uncoupled cortical areas. The frequencies are measured from the Fourier transform of the cross-correlograms. (c): the signal-to-noise for two different situations of the cortico-cortical interaction: when thy are coupled (circles) and when they are uncoupled (triangles). In panel $(\mathrm{d})$ we plot the signal-to-noise ratio for an increasing value of the cortico-cortical interaction strength for different values of $\nu_{T}$.

the local maximum the signal-to-noise increases again monotonically for very large values of the rate $\nu_{T}$. The signal-to-noise ratio as a function of the strength of the cortico-cortical connection for different values of $\nu_{T} / \nu_{0}$ is illustrated in Fig. 5d. Interestingly, for low values of $\nu_{T} / \nu_{0}$ the signal-to-noise response is flat but increases for large $C_{C C}$ while it is flat but decreases for higher values of $\nu_{T} / \nu_{0}$.

Effect of the cortico-cortical connection. The mean firing rate $F$ of the three neuronal populations as a function of the strength $C_{C C}$ at an input level $\nu_{T}=7 / 3 \nu_{0}$ is 

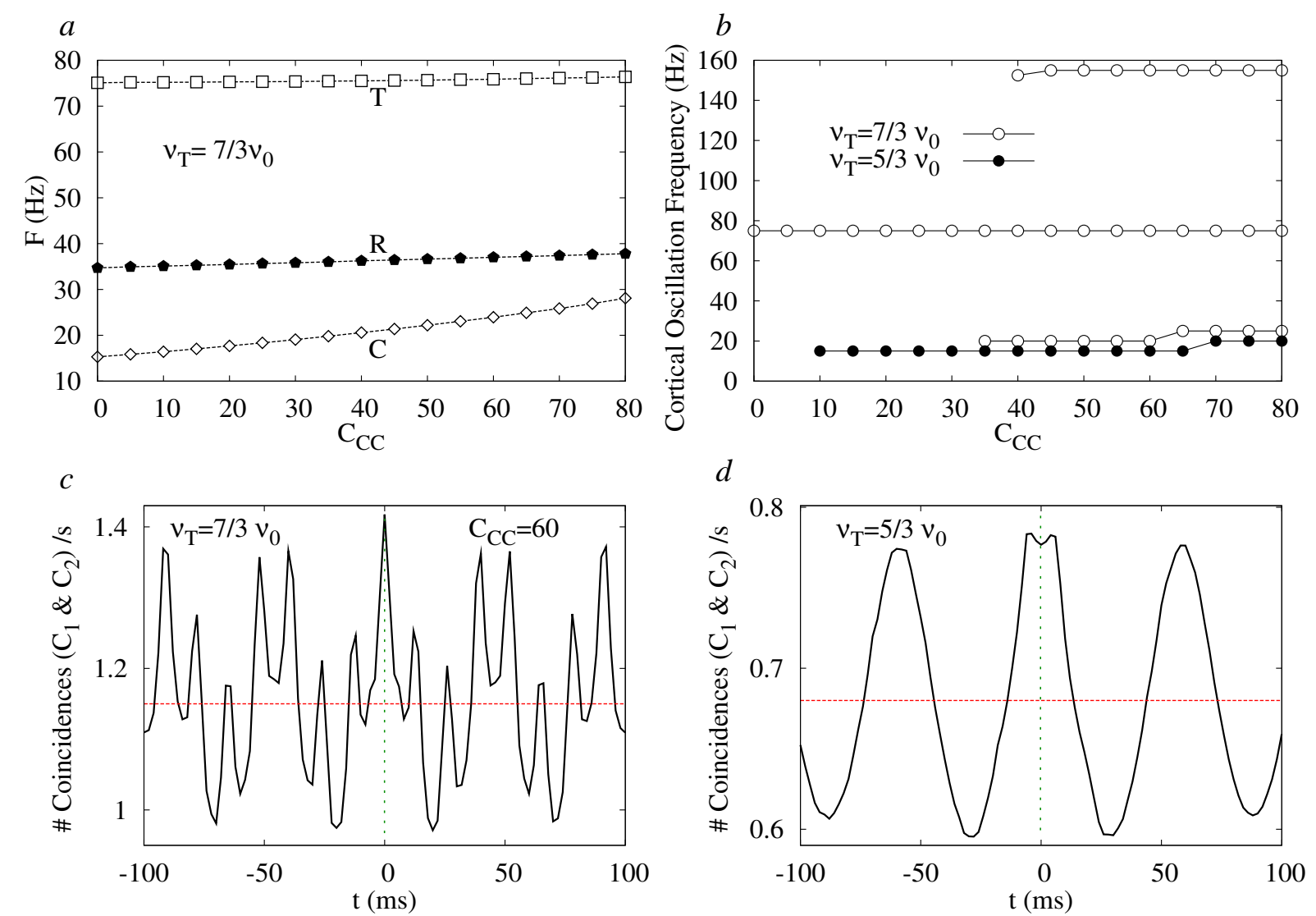

FIG. 6: (color online) Effect of the cortico-cortical connection. Panel (a): firing rate of the T, R and $\mathrm{C}$ areas as a function of the cortico-cortical interaction strength for $\nu_{T}=7 / 3 \nu_{0}$. Panel (b) cortical oscillation frequency vs. $C_{C C}$ for two different values of $\nu_{T}\left(5 / 3 \nu_{0} ; 7 / 3 \nu_{0}\right)$. Panel (c): Cross-correlogram between $C_{1}$ and $C_{2}$ areas for $C_{C C}=60$ and $\nu_{T}=7 / 3 \nu_{0}$, the local maximun closest to zero are located at $\pm 12 \mathrm{~ms}$. Panel (d): same as panel (c) but for $\nu_{T}=5 / 3 \nu_{0}$, and the maximun are not exactly at zero-lag but at $\pm 6 \mathrm{~ms}$.

illustrated in Fig. 6a. This figure shows that the cortical firing rate is indeed the most affected rate and increases monotonically with an increase in the cortico-cortical connectivity. The dominant frequencies of cortical oscillations determined by the power spectrum analysis are displayed in Fig. 6b as a function of cortical connectivity and for two levels of external input to the thalamus. For a value $\nu_{T}=5 / 3 \nu_{0}$ a single frequency appears almost constant and independent of the $C_{C C}$ strength. On the contrary, at $\nu_{T}=7 / 3 \nu_{0}$ three frequency components appear for $C_{C C}>35$. Like in Fig $5 \mathrm{~b}$ the lowest frequency is associated to the cortical firing rate and the intermediate frequency is associated to the firing rate of population $\mathrm{T}$. The highest frequency could also be associated to the inverse of the delay 

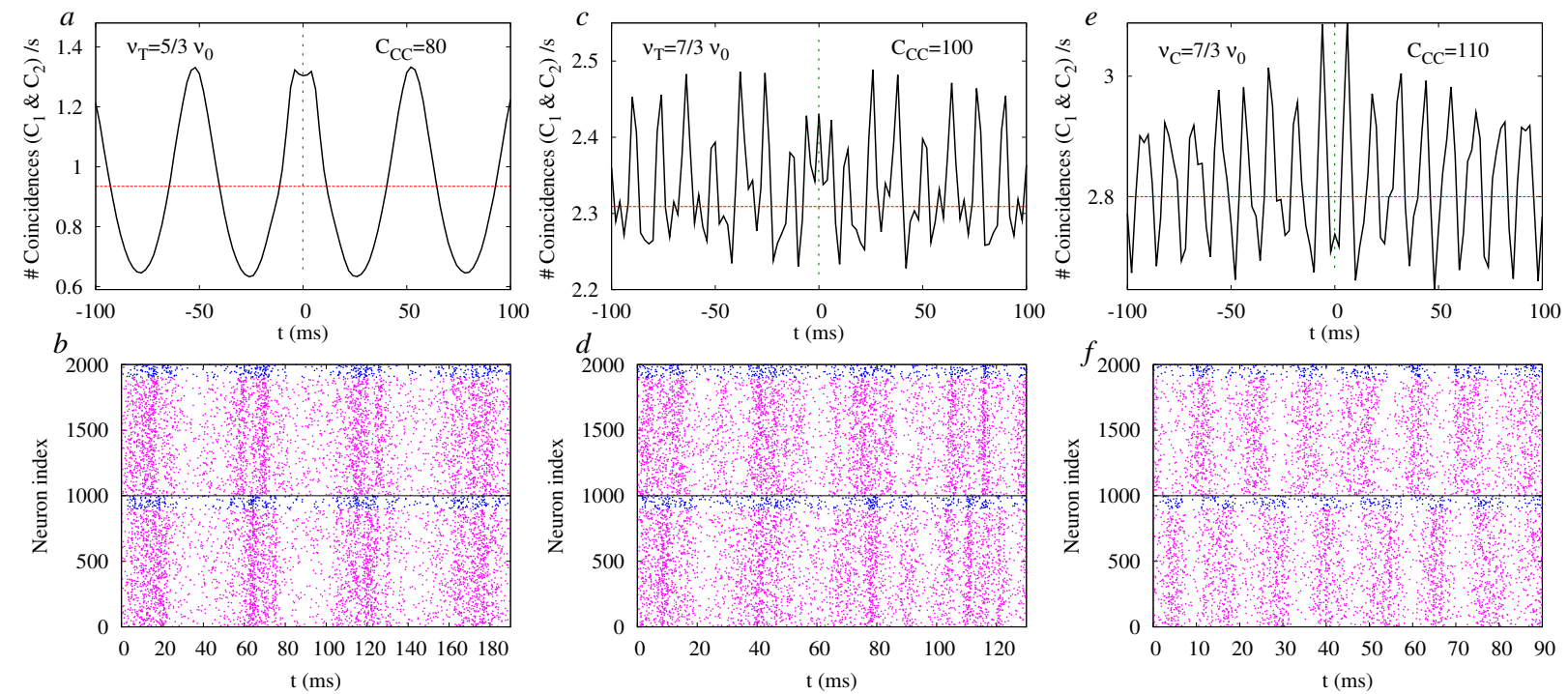

FIG. 7: (Color on line) Dynamics of the cortical area as a function of the cortico-cortical interaction strength. Panel (a): cross-correlogram for $\nu_{T}=5 / 3 \nu_{0}$ and $c_{c c}=80$; Panel B: corresponding raster plot of all the cortical neurons in both cortical areas. Panel (c): cross-correlogram for $\nu_{T}=7 / 3 \nu_{0}$ and $c_{c c}=100$; Panel $(\mathrm{d})$ : corresponding raster plot. Panel (e): cross-correlogram for $\nu_{T}=7 / 3 \nu_{0}$ and $c_{c c}=110 ;$ Panel (f): corresponding raster plot.

time in the cortico-cortical connection and became more important for higher values of $C_{C C}$. The presence of multiple oscillatory frequencies can be clearly observed in the crosscorrelogram for $C_{C C}=60$ and $\nu_{T}=7 / 3 \nu_{0}$ (Fig. 6c), whereas a single frequency component dominates the dynamics for $\nu_{T}=5 / 3 \nu_{0}$ (Fig. 6d).

The observation of the raster plots and of the cross-correlograms illustrates further the dynamics emerging from the interaction between the cortical areas. In Fig. 7a, b it can be observed that for $C_{C C}=60$ and $\nu_{T}=5 / 3 \nu_{0}$ the slow frequency component related to the cortical firing frequency is predominant. The peak is not sharp, at $\pm 4 \mathrm{~ms}$ from the zero-lag, and a "master-slave" dynamics can be observed in the region of high instantaneous firing rate (say from 50-80 ms after the external input onset). With parameters of $C_{C C}=$ 100 and $\nu_{T}=7 / 3 \nu_{0}$ multiple frequencies are observed in the raster plot and in the crosscorrelogram (Fig. 7c,d). In this case, both the zero-lag cortical synchronization and the leader-ladder dynamics present a strong competition. At very large values $C_{C C}=110$ the cortico-cortical connection dominates and gives rise to an out-of-phase cortical synchronized dynamics between the two areas (Fig. 7e,f) The signature of this dynamics appears both 
in a double peak at $\pm 6 \mathrm{~ms}$ (corresponding to the cortico-cortical coupling time in the cross correlation function) and in the raster plot where zero-phase synchronization does not occur between the cortical areas.

\section{Discussion}

We have presented the dynamics of a simplified thalamocortical circuit. Our results suggest that the thalamus could be a central subcortical area that is able to trigger the emergence of zero-lag synchrony between distant cortical areas due to a dynamical relaying $[1,33]$. According to this phenomenon a central element can enable two populations to synchronize at zero-lag. Other subcortical areas such as the brainstem [34] and the hippocampus are likely to play a similar role in dynamical relaying. However, the peculiar recurrent connections of the thalamic reticular nucleus $[15,16]$ might provide the thalamocortical circuit with specific features that do not account just for the synchronized pattern, but also for switching "on" or "off" the asynchronous state. Furthermore, considering that large scale integration may occur as a consequence of neuronal coherence, the critical question about how the dynamical selection of integrated areas is achieved remains open $[1,11,35,36]$. We suggest that an increase in the external activity fed into the $\mathrm{T}$ population with respect to that of $\mathrm{R}$ yields the cortical areas synchronize at zero-phase lag as depicted in Fig 3. That means the thalamus would be able to control the cortical synchronous state and regulate large scale integration. This control can occur at a fast time scale in agreement with experimental data and without any need of plasticity or adaptation mechanisms which typically require longer time scales. The main input sources to $\mathrm{T}$ are the ascending sensory input and the descending cortico-fugal pathway, thus suggesting that both inputs may play an important role in controlling cortical synchrony. This hypothesis for the cortico-petal projections is complementary to the hypothesis of "adaptive filtering" suggested elsewhere for the cortico-fugal projections [18, 22, 23].

According to our model, see Figs. 5b,6b, the thalamocortical circuit is able to generate fast oscillations in frequency ranges like beta and gamma bands triggered by an external input to the thalamus formed by independent Poisson trains. The question of how to generate such fast oscillations has been largely discussed in the literature[14, 37-41] but, as recently pointed out [26], empirical phenomena like the cycle skipping were not satisfactorily described. The 
cycle skipping is observed experimentally in the current thalamocortical model when each cortical neuron spikes according to a gamma frequency modulation but with a smaller firing rate. In the raster plots of Fig. $3 \mathrm{a}$ it is possible to observe that few neurons spike at a given gamma cycle. Then, the oscillations are in fact shared by a whole population while single neurons skip cycles. As shown in Figs. 5a, b the cortical oscillations, for instance at a signal-to-noise ratio local maximun $\nu_{T} \simeq \frac{7}{3} \nu_{0}$, occur at frequencies near $80 \mathrm{~Hz}$ for disconnected areas and in multiple frequencies for $C_{C C}=40$, while the average firing rate is approximately $\frac{1}{4}$ of it, 20 spikes/s. In general, the firing rate of the cortical populations (see Figs. 5a,6a) were found to be related to the lowest frequency component in case of multiple frequency oscillations. Otherwise the cortical firing rate tends to be much lower than the single frequency of oscillations (e.g., Fig. 5b) or close to it for low external driving $\left(\nu_{T}=\frac{5}{3} \nu_{0}\right)$ (e.g., Fig. 6b).

The current results emphasize the hypothesis that the thalamus could control the dynamics of the thalamocortical functional networks enabling two separated cortical areas to be either synchronized (at zero-lag) or unsynchronized. Correlations in the output firing rate of two neurons have been shown to increase with the firing rate [42]. Indeed we observed that for increasing input rates $\left(\nu_{T}\right)$ the firing rate of all populations increase monotonically, accordingly to an expected sigmoidal function (Fig. 5a). König and collaborators [43] reported physiological evidence of long-range synchrony with oscillations, whereas short-range synchrony may occur with or without oscillations. Our results, especially for small corticocortical connectivity (say smaller than the internal conectivity), are in agreement with this finding. However, synchrony without oscillations in local circuit may appear due to extensive sharing of common excitatory inputs which typically generate the zero-lag coincidence observed when neurons are fire at high rates [42]. Conversely, neurons correlated by longrange connections are likely to share very few synaptic driving, such that synchrony without oscillations should be very rare.

In order to suggest an insight of the model with the anatomical pattern of the circuit one should consider that the thalamocortical and corticothalamic projections are reciprocal to a great extent but corticothalamic projections are characterized by a dual pattern of synaptic morphology [44]. The modal switch of corticothalamic giant synapses controlled by background activity was recently reported [45]. We speculate that considering this finding and our results together we may suggest that the dual pattern of corticothalamic synapses 
might correspond to a dual function: one synaptic type involved in assessing the circuitry necessary for the build-up of cortico-cortical synchronization, somehow a non-contextual cueing system, and the other synaptic type involved in carrying contextual cueing. which is a question that the current study is unable to answer. We must also consider the fact that our model of individual dynamics of the integrate-and-fire neurons does not produce burst discharges $[46,47]$. This is a clear limitation and the inclusion of a more physiologically realistic model as well as greater neuronal diversity [48] are scheduled for our future work. Despite the oversimplification of our circuitry and the neural network modeling in general the robustness of our model is an interesting outcome of this study. We have arbitrarily kept the external input $\nu_{0}$ over $\mathrm{R}$ and the cortex populations fixed but we might have kept fixed $\mathrm{T}$ and the cortex populations with a variable external input into $\mathrm{R}\left(\nu_{R}\right)$. In fact it is the dependency on the variable $\frac{\nu_{T}}{\nu_{R}}$ which represents the control key of the dynamic activity of the system as both rates of external inputs $\left(\nu_{T}, \nu_{R}\right)$ are varying over time $[49,50]$. The importance of uncorrelated inputs can be viewed as emphasizing the role of so-called "background activity", which was already reported to play an important role in controlling the thalamocortical circuit dynamic state [51]. We are convinced that further simulations with more accurate details of the neuronal models and with embedded models of the dual corticofugal connectivity may provide critical clues for better understanding the mechanisms of the dynamical control subserving the synchronization of cortico-cortical distributed activity.

\section{Acknowledgments}

We thank Raul Vicente and Javier Iglesias for useful comments and suggestions. The authors acknowledge financial support from the European Commission Project GABA (FP6NEST Contract 043309), LLG and CM also acknowledge the MEC (Spain) and Feder under project FIS2007-60327 (FISICOS).

[1] R. Vicente, L. L. Gollo, C. R. Mirasso, I. Fischer, and G. Pipa, Proc. Natl. Acad. Sci. 105, 7157 (2008).

[2] L. L. Gollo, O. Kinouchi, and M. Copelli, PLoS Comput Biol 5, e1000402 (2009).

[3] A. E. P. Villa, I. V. Tetko, B. Hyland, and N. A., Proc. Natl. Acad. Sci. 96, 1006 (1999). 
[4] G. Hayon, M. Abeles, and D. Lehmann, J. Comp. Neurosci. 18, 41 (2005).

[5] P. Fries, D. Nikolić, and W. Singer, Trends Neurosci. 30, 309 (2007).

[6] C. M. Gray, P. König, A. K. Engel, and W. Singer, Nature 338, 334 (1989).

[7] A. Engel, Kreiter, A.K., P. Konig, and W. Singer, Proc. Natl. Acad. Sci. 88, 6048 (1991).

[8] M. Castelo-Branco, R. Goebel, S. Neuenschwander, and W. Singer, Nature 405, 685 (2000).

[9] P. Tiesinga, J. Fellows, and T. Sejnowski, Nature Rev. Neuroscience 9, 97 (2008).

[10] C. von der Marlsburg, Kybernetic 14, 85 (1973).

[11] P. Fries, Trends in Cognitive Sciences 9, 474 (2005).

[12] G. Desbordes, J. Jin, C. Weng, N. A. Lesica, G. B. Stanley, and J.-M. Alonso, PLoS Biol 6, e324 (2008).

[13] D. Contreras, A. Destexhe, T. Sejnowski, and M. Steriade, Science 274, 771 (1996).

[14] R. D. Traub, M. A. Whittington, I. M. Stanford, and J. G. R. Jefferys, Nature 383, 621 (1996).

[15] E. G. Jones, The Thalamus (Plenum Press, New York, 1985).

[16] S. M. Sherman, Prog Brain Res. 149, 107 (2005).

[17] M. Steriade and R. R. Llinas, Physiological Review 68, 649 (1988).

[18] A. E. P. Villa, I. V. Tetko, P. Dutoit, Y. De Ribaupierre, and F. De Ribaupierre, J. Neurosci. Meth. 86, 161 (1999).

[19] A. E. P. Villa, in Virtual lesions: Examining Cortical Function with reversible Deactivation, edited by S. G. Lomber and R. A. W. Galuske (Oxford University Press, Oxford, UK, 2002), chap. 4, pp. 83-119.

[20] D. McCormick and T. Bal, Current Opinion in Neurobiology 4, 550 (1994).

[21] A. E. P. Villa, V. M. Bajo, and G. Vantini, Brain Research Bulletin 39, 139 (1996).

[22] A. E. P. Villa, E. M. Rouiller, G. M. Simm, P. Zurita, Y. de Ribaupierre, and F. de Ribaupierre, Exp. Brain Res. 86, 506 (1991).

[23] I. V. Tetko and A. E. P. Villa, Neural Networks 10, 1361 (1997).

[24] J. L. Ringo, R. W. Doty, S. Demeter, and P. Y. Simard, Cerebral Cortex 4, 331 (1994).

[25] R. Vicente, L. L. Gollo, C. Mirasso, I. Fischer, and G. Pipa (2009), in press.

[26] D. Nikolić, in Proceedings of the International Joint Conference on Neural Networks, IJCNN (2009).

[27] R. Milo, S. Shen-Orr, S. Itzkovitz, N. Kashtan, D. Chklovskii, and U. Alon, Science 298, 824 
(2002).

[28] R. Brette and et al., Journal of Computational Neuroscience 23, 349 (2007).

[29] J. M. Eppler, M. Helias, E. Muller, M. Diesmann, and M. Gewaltig, Front. Neuroinform. 2, $12(2009)$.

[30] N. Brunel, Journal of Computational Neuroscience 8, 183 (2000).

[31] G. M. Shepherd, ed., The Synaptic Organization of the Brain (Oxford University Press, 1998).

[32] J. R. Huguenard and D. A. McCormick, Trends in Neurosciences 30, 350 (2007).

[33] I. Fischer, R. Vicente, J. M. Buldu, M. Peil, C. R. Mirasso, M. C. Torrent, and J. GarciaOjalvo, Physical Review Letters 97, 123902 (2006).

[34] B. Scheller, M. Daunderer, and G. Pipa, Anesthesiology 111, 340 (2009).

[35] E. Salinas and T. Sejnowski, Nat. Rev. Neurosci. 2, 539 (2001).

[36] P. Uhlhaas, G. Pipa, B. Lima, L. Melloni, S. Neuenschwander, D. Nikolić, and W. Singer, Front. Integr. Neurosci. 3 (2009).

[37] B. Doiron, M. J. Chacron, L. Maler, A. Longtin, and J. Bastian, Nature 421, 539 (2003).

[38] B. Doiron, B. Lindner, A. Longtin, L. Maler, and J. Bastian, Phys. Rev. Lett. 93, 048101 (2004).

[39] C. Börgers, S. Epstein, and N. J. Kopell, Proceedings of the National Academy of Sciences of the United States of America 102, 7002 (2005).

[40] D. Marinazzo, H. J. Kappen, and S. C. A. M. Gielen, Neural Computation 19, 1739 (2007).

[41] C. Börgers, S. Epstein, and N. J. Kopell, Proceedings of the National Academy of Sciences 105, 18023 (2008).

[42] J. de la Rocha, B. Doiron, E. Shea-Brown, K. Josic, and A. Reyes, Nature 448, 802 (2007).

[43] P. König, A. Engel, and W. Singer, Proc Natl Acad Sci USA 92, 290 (1995).

[44] M. Takayanagi and H. Ojima, Neuroscience 142, 769 (2006).

[45] A. Groh, C. P. J. de Kock, V. C. Wimmer, B. Sakmann, and T. Kuner, Journal of Neuroscience 8, 9652 (2008).

[46] S. M. Sherman, Trends Neurosci. 24, 122 (2001).

[47] R. Krahe and F. Gabbiani, Nat Rev Neurosci. 5, 13 (2004).

[48] C. I. Buia and P. H. Tiesinga, J Neurophysiol 99, 2158 (2008).

[49] K. McAlonan, J. Cavanaugh, and R. H. Wurtz, Nature 456, 391 (2008).

[50] X. Yu, X. Xu, S. He, and J. He, Nat Neurosci 12, 1165 (2009). 
[51] J. Wolfart, D. Debay, G. Masson, A. Destexhe, and T. Bal, Nature Neuroscience 8, 1760 (2005). 